Preference for Hierarchy is Related to the Motivation to Feel Less Empathy and More Schadenfreude Towards Low-Status People

Word Count: 9788 


\begin{abstract}
Social dominance orientation (SDO) — the tendency to accept and endorse group-based dominance_-has been linked with reduced empathy and increased schadenfreude (i.e., pleasure at the misfortunes of others) towards competitive others. Are these outcomes driven by a strategic motivation to feel emotions that facilitate hierarchy-reinforcing behaviors (and avoid those that interfere)? Across three preregistered studies using Amazon Mechanical Turk participants $(N=1724)$, we find that SDO is associated with which emotions people want and choose to feel. People with higher (relative to lower) levels of SDO make similar predictions of others' emotions when asked, but desire to feel less empathy and more schadenfreude toward low-status targets, and when given a choice, choose to feel less empathy and more schadenfreude. This work adds to a growing literature on the impact of ideology_in this case, SDO-on emotion tendencies and further expands work on the motivated nature of empathy.
\end{abstract}

Keywords: social dominance orientation, empathy, schadenfreude, motivated cognition 


\section{Preference for Hierarchy is Related to the Motivation to Feel Less Empathy and More Schadenfreude Towards Low-Status People}

Social dominance orientation (SDO; Ho et al., 2015), or the extent to which individuals accept and promote group-based inequality, reflects a competitive world view and is a potent predictor of attitudes and behaviors. For example, SDO positively relates to hierarchy-enhancing attitudes such as racism and sexism (Hiel \& Mervielde, 2005; Sibley et al., 2007), as well as support for anti-affirmative action, and strict immigration policies (Craig \& Richeson, 2014; Haley \& Sidanius, 2006). Although there is a rich literature on SDO's influence on individuals' attitudes and behaviors, there is surprisingly little on the relationship between SDO and emotions. Emotions represent reactions to specific targets, situations, and contexts that go beyond positive and negative evaluations that are captured when assessing attitudes (Cottrell \& Neuberg, 2005; Mackie et al., 2000). Emotions are often better predictors of behavior than attitudes due to this representational richness (Dovidio et al., 2010).

One exception is the work examining the relationship between SDO, empathy, and counter-empathy, two related social emotions that are well-studied within intergroup conflicts specifically. SDO has been associated with lower levels of trait empathy, measured as empathic concern and/or perspective taking (Nicol \& Rounding, 2013; Sherman et al., 2015; Sidanius et al., 2013), in both cross-sectional and longitudinal research. Additionally, SDO is associated with reduced levels of state empathy - the congruent emotional reaction a person feels in response to the assumed emotional state of others (Cikara et al., 2014; Lucas \& Kteily, 2018) - and increased levels of state counter-empathy, the opposite emotional reaction in response (Hudson et al., 2019). 
Counter-empathy has two facets: schadenfreude, or feeling positively in response to another person's pain, and gluckschmerz, or feeling negatively about another person's pleasure (Smith \& van Dijk, 2018).

Although empathy is often regarded as an automatic process, individuals are motivated to feel or avoid empathy depending on the target and the context (Zaki, 2014). Specifically, people tend to feel less empathy and more counter-empathy towards out-groups and in competitive settings, which is posited to serve an adaptive function to foster in-group cohesion and out-group exclusion (Cikara et al., 2011). Similarly, schadenfreude is theorized to serve as a dominance social-functional regulator (Lange \& Boecker, 2019), employed towards individuals in order to lower their perceived dominance in the eyes of others. Thus, a 'motivated (counter-)empathy' perspective generates the prediction that feeling empathy, and perhaps not feeling counter-empathy, towards competitive out-groups is antithetical to establishing groupbased dominance. In other words, the competitive, hierarchically-oriented nature of those higher in SDO would make it functional for them to feel less empathy and perhaps more counter-empathy, as feeling those emotions help reify the social hierarchies at stake.

That said, SDO reflects a proclivity towards establishing and protecting groupbased hierarchies, and therefore is overall positively related to attitudes, policies, and behaviors that enhance and entrench hierarchy and negatively related to things that would attenuate it. If one's goal is to maintain hierarchy, it would be counter-productive to feel reduced empathy and increased counter-empathy towards in-group members as well as high-status groups, as feeling these emotions would destabilize the hierarchy. 
Indeed, manipulating the status of targets can lead to a reversal of the SDO - empathy link; SDO can be positively related to empathy, but specifically for high-status targets (Lucas \& Kteily, 2018) and not low-status ones.

These findings parallel work on SDO and attitudes. For example, SDO is generally associated with anti-affirmative action support (Federico \& Sidanius, 2002) but can be positively related if such support supports racial, gender, and socioeconomic hierarchies (Fraser et al., 2015; Gutiérrez \& Unzueta, 2013; G. C. Ho \& Unzueta, 2015). As another example, SDO predicts increased and decreased support for immigration when the immigrants in question are high and low-status, respectively (Pratto \& Lemieux, 2001; Thomsen et al., 2008). Finally, SDO is usually correlated with a diminished interest in the morality of policies, but that disinterest is only reserved for policies that harm low-status groups (Lucas \& Kteily, 2016).

In sum, SDO's relationship with affect, cognition, and behavior should depend on whether having a given set of emotions/attitudes or engaging in a given set of behaviors will create and maintain hierarchy (irrespective of one's personal position in the hierarchy). Consequently, the amounts of empathy and counter-empathy elicited by intergroup contexts are not only correlated with levels of SDO but motivated by it. This proposition dovetails with recent research on the impact of holding certain political and economic ideologies on emotional regulation and expression. For example, believing that the current economic system is justified (i.e., system justification; Jost et al., 2004) is correlated with reduced emotional response to evidence of poverty and income inequality (Goudarzi et al., 2019). Other work shows that political ideology motivates people's desire to feel group based emotions like anger and empathy towards 
outgroups, which in turn predict political reactions (Hasson et al., 2018; Jost et al., 2003; Porat et al., 2016). Thus, this work seeks to add to the rapidly growing literature on the impact of ideology on intergroup emotions.

\section{Current Research}

Our overarching hypothesis is that the link between SDO and the desire to experience (counter-)empathy is context and target-dependent: strengthening when feeling a given emotion leads to hierarchy maintenance and weakening when the emotion leads to hierarchy attenuation. More specifically, high-SDO people should want to feel empathy (and not counter-empathy) toward high-status targets because that pattern of emotions helps maintain the hierarchy, whereas high empathy/low counterempathy towards low-status targets works against the hierarchy.

Given SDO's negative relationship with trait levels of empathic concern and perspective taking (e.g., Sidanius et al., 2013), there is a possibility that SDO reflects a reduced ability to perceive the emotional states of others, rather than SDO motivating the predicted pattern of emotional responses. Study 1 sought to rule out the possibility that the relationship between SDO and (counter-) empathy simply reflects a tendency among high-SDO individuals to ascribe fewer extreme emotions to others (which could then account for their own blunted emotional responses).

After establishing that SDO does not correlate with respondents' ability to perceive emotions, Study 2 assessed whether SDO is associated with individuals' selfreported desire to feel reduced empathy and increased schadenfreude towards others. Previous work on political orientation found differences in the amount of empathy individuals wanted to feel towards political outgroups compared to the amount of 
empathy they actually felt (Hasson et al., 2018). We extend that work by investigating SDO as the ideology motivating empathy, as well as assessing levels of counterempathy, schadenfreude specifically. Finally, Study 3 indexes motivation via behavior, by assessing individuals' desire to approach or avoid situations in which they were asked to feel empathy or schadenfreude.

As we have noted above, we predict that our findings will be target-dependent. Extending previous work (Cikara \& Fiske, 2013), we used targets borrowed from the Stereotype Content Model (Cuddy et al., 2008) to investigate whether target status moderates SDO's relationship with empathy and schadenfreude. Individuals and groups that are stereotyped to be high-status (and low in cooperation) are often targets of low levels of empathy and high levels of schadenfreude when misfortunes befall them. However, these are often the very individuals and groups at the top of the social hierarchy. Thus, we would expect individuals high in SDO to feel more empathy (and less schadenfreude) towards targets that are high-status compared to low-status targets. Furthermore, we predict the same moderation by target status for participants' desire to feel these emotions and their choice of behavior. A priori we did not have a clear prediction regarding the interaction of participant SDO and target cooperation (but see the general discussion section). Thus, another aim of the current work was to investigate the role of target cooperation. As such we employed a fully crossed design, including targets that were high and low on both the cooperation and status dimensions.

All studies were pre-registered and the entire project, including de-identified data, supplementary materials and analyses, can be found on OSF at https://osf.io/4p9sg/?view_only=d83a33e1f8984037bd0fa240b91566ef. In these 
studies, we report all measures, manipulations, and exclusions and our sample sizes were determined before any data analysis. Studies 1-3 preregistrations can be found at the following links:

https://osf.io/y54a7/?view_only=8fa4b3e27b5d4169a212b7b545053191;

https://osf.io/b7h5a/?view_only=3c6b7980cc2d4e69b6ea2c06f427c905; https://osf.io/2agyn/?view_only=1cd6d18b6cca4357a8ab0f8cd47e4426.

Study 1: The relationship between SDO and the tendency to attribute emotion versus feel empathy and schadenfreude

In Study 1 we examined SDO's relationship with the tendency to infer others' emotional states, as such a tendency is a precursor for the type of motivated affective empathic and counter-empathic responses we are hypothesizing. We randomly assigned participants to either A, report their levels of empathy and schadenfreude towards targets, varied in status and cooperation, that suffered a mild misfortune or B, report how they believe the target felt in the situation. The latter condition did not measure participants' levels of empathy and schadenfreude but instead measured how well participants understood the emotional state of another person. By assessing emotional forecasts between-subjects we were able to reduce carry-over effects that can occur within subjects. Finally, Study 1 expands upon previous work concerning SDO and (counter-)empathy by investigating third-party status hierarchies instead of group membership.

We had three hypotheses. First, we expected that participants assigned to report how the target felt in the situations would indicate that the target felt more negatively than positively. More importantly, we hypothesized that this effect would not be 
moderated by the status or cooperation of the target, as well as not moderated by participants' levels of SDO. Second, we expected to replicate past work on empathy and schadenfreude for SCM targets such that participants would report the highest levels of empathy for high-cooperation/low-status targets and the highest levels of schadenfreude for low-cooperation/high-status targets (Cikara \& Fiske, 2011, 2012). Third and finally, we expected that SDO would moderate the above effect, such that as SDO increased, participants would report relatively more empathy and less schadenfreude for the high-status targets as compared to the low-status targets.

\section{Method}

\section{Participants}

Our sample size estimates were based on recommendations by Schönbrodt and Perugini (2013) in which correlations of $r=.21$ (the average $r$ effect size in psychology; Richard, Bond, \& Stokes-Zoota, 2003) stabilize at $\sim 250$ subjects. Given we had a between-subjects variable with two levels, we doubled 250 for a planned sample size of 500 and recruited 50 more subjects to allow for attrition. We paid a total of 552 participants from Amazon Mechanical Turk using Turk Prime as a recruitment platform, restricting our potential sample to those who were 18 years or older, residing in the United States, and had completed 1000 or fewer hits. Before participants reached the consent form, they were screened for potential bots and lack of attention. Participants who failed to enter the correct answer never completed any study measures and were not paid. We further excluded 76 participants who failed another attention check embedded within the Emotion Rating task (described below), leaving a final sample of 476 participants (332 self-identified Whites and 245 women), $M_{\text {age }}=33.26, S D=10.77$. 
We ran a one sample Chi-square test to assess whether there was a significant difference in the number of participants in each condition described below. There were an equal number of participants in the two conditions ( $n_{\text {Self-Focus }}=242, n_{\text {Target-Focus }}=$ 234), $\chi^{2}=0.13, p=.714$.

\section{Materials}

Emotion Rating Task. Participants read a series of nine stories purportedly written by previous participants about their recent experiences. After each story, participants either answered how good and bad they themselves felt (Self-Focus; schadenfreude and empathy respectively) or how good and bad they believed the target in the story felt (Target-Focus). Specifically, in the Target-Focus condition, participants responded to the prompt "If you saw this scenario in real life, how good/bad do you think [TARGET NAME] feels?" while participants in the Self-Focus condition responded to "If you saw this scenario in real life, how good/bad would it make you feel?" Participants indicated good and bad feelings on 100-point sliders anchored at Not at All Good/Bad (0) to Extremely Good/Bad (100) without feedback. The order of the good/bad sliders were randomized between trials.

One potential concern is that asking participants about how "good" and "bad" they felt about a negative event befalling another target did not accurately, or adequately, capture schadenfreude and empathy respectively. However, factor analysis of a multi-item empathy and schadenfreude measures suggested that "how good" and "how bad" are good proxies for these emotions (Cikara et al., 2014; Hudson et al., 2019). More specifically, out of the items that loaded onto the empathy factor (i.e., sympathetic, sad, compassionate, and concerned), "how bad" loaded the strongest 
(range $=.89-.56)$. Similarly, out of the items that loaded onto the schadenfreude factor (i.e., relieved, happy, and satisfied), "how good" loaded the strongest (range $=.94-$ .88). Furthermore, we asked about how participants felt about the event that happened to the target, not how they feel about the target specifically.

Within each trial, participants learned the name of the target, a descriptor of the target corresponded to one of the four dimensions in the SCM, and a negative event that happened to the target. We chose the target descriptor based on past SCM work (Fiske et al., 2002): "a drug addict" and "a homeless man" represented low-status and low-cooperation; "a blind man" and "an 80-year old man" represented low-status and high-cooperation; "an investment banker" and "a venture capitalist" represented highstatus and low-cooperation; and "a firefighter" and "a honor college student" represented high-status and high-cooperation. Within a given trial, the name, target, and story were randomly combined. We included one attention check trial in which participants were instructed to slide both sliders all the way to the left (i.e., "mark good and bad feelings as 'zero'”). Given that the sliders did not give numerical feedback (meaning participants did not know if they actually slid the sliders all the way to 0), we included all participants where both sliders were valued at less than 5 for the attention check.

Warmth and Competence Ratings. According to the SCM, status predicts attributions of competence and cooperation predicts attributions of warmth. For each of the eight targets we asked participants to indicate how warm (i.e., good-natured, sincere, friendly; Extremely Cold - Extremely Warm) and competent (i.e., confident, capable, skillful; Extremely Incompetent - Extremely Competent) they were on 100- 
point sliders. We asked the warmth and competence questions sequentially by target, with a single target and the two sliders presented on a page at any time. We counterbalanced the order of the warmth and competence sliders within a target as well as the presentation order of all targets. In the aggregate, the relative positions of the targets to each other were in line with SCM predictions (see supplementary materials).

Social Dominance Orientation. We used the shortened eight-item $\mathrm{SDO}_{7}$ scale to measure social dominance on a 1 (Strongly Oppose) - 7 (Strongly Favor) scale, creating a single index, $\alpha=.88, M=2.56, S D=1.18$. Higher numbers indicated a higher preference and support for group-based inequality. Sample items included "We should do what we can to equalize conditions for different groups (reverse-coded)" and "Some groups of people are simply inferior to other groups." We tested whether SDO levels varied as a function of the Emotional Focus condition. They did not, $F(1,474)=1.04, p$ $=.308$. SDO also did not vary as a function of the counter-balancing order with the warmth and competence ratings, $F(1,474)=1.79, p=.182$.

\section{Procedure}

Participants who passed an initial attention check completed an Emotion Rating task in which they read a series of stories about negative events that happened to target individuals. We randomly assigned participants to one of the two (Emotional Focus) conditions; some participants indicated the purported emotions of the target in the stories (Target-Focus) while other participants indicated how they themselves felt in response (Self-Focus). After the Emotion Rating task, participants rated the targets in the stories on warmth and competence and filled out a shortened $\mathrm{SDO}_{7}$ questionnaire. 
We counterbalanced the order of the warmth/competence ratings and the SDO questionnaire.

\section{Analyses $^{1}$}

We pre-registered our data analysis plan, in which we ran a multilevel hierarchical model in $R$ where emotion ratings were predicted by the five-way interaction between Emotional Focus (Self-Focus, Target-Focus), Target Status (High, Low), Target Cooperation (High, Low), Rating ("How Good", "How Bad"), and SDO (meancentered), including a participant random intercept. All variables were within-subjects except for Emotional Focus, which was between-subjects. Variables were effects-coded with Target-Focus, High Status, High Cooperation, and "How Bad" as reference categories. We built the model hierarchically from the random effects model to the fiveway interaction model. At each step until the four-way model, the more complicated model was a better predictor of the data. However, the five-way model was a marginally better model than the three-way model $(p=.056)$ and was a significantly better model than the four-way model $(p=.015)$. Furthermore, we pre-registered that we would report statistics from the fully saturated model, which is what we do below.

We calculated a pseudo- $\mathrm{R}^{2}$ for multilevel models using the "r.squaredGLMM" function from the MuMln package (Bartoń, 2018). The marginal $R^{2}$ of the five-way model (or the variance explained by fixed factors) was .43 (the conditional $R^{2}=.45$ ). We also conducted sensitivity analyses using the SIMR package to determine whether we had sufficient power to test these interactions. With $80 \%$ power we could have detected

\footnotetext{
${ }^{1}$ We pre-registered that we would run a version of the model in which we replaced our a priori cooperation/status target designations with the continuous ratings participants gave regarding the targets' warmth and competence. These analyses can be found in supplementary materials for Studies 1 and 2 .
} 
an effect size as small as 0.61 . We used the emmeans package in $R$ to conduct planned comparisons between means and slopes, which were adjusted using the Tukey method. All descriptive statistics, correlations, ANOVA, and regression analyses tables not found in the main text can be found in supplementary materials.

\section{Results}

\section{Comprehension Check}

As predicted, the two-way interaction between Emotional Focus and Rating (i.e., whether it was the 'good' or 'bad' slider) was significant, $F(1,7111.12)=192.27, p<$ .001. When participants judged the emotions of targets experiencing negative events, they believed that the target felt more bad $(M=67.06)$ than good $(M=25.08)$ in the scenario $t(7111)=-59.69, p<.001, d=-2.06$. We hypothesized that Target Status, Target Cooperation, and SDO would not moderate this relationship. That hypothesis was not supported, as all three variables significantly interacted with Rating and Emotional Focus. Participants assumed that the high cooperation target $(M=66.02)$ felt less bad than the low cooperation target $(M=68.10), t(7111)=2.10, p=.036, d=0.09$ and that the high status target felt less bad $(M=64.82), t(7111)=-4.51 p<.001, d=-$ 0.20 , and more good $(M=27.03), t(7111)=3.91, p<.001, d=0.22$, in the scenario than the low status target $\left(M_{\text {bad }}=69.30, M_{\text {good }}=23.14\right)$.

\section{Did social dominants differentially attribute emotions to others in general?}

Yes, but narrowly. SDO interacted with Emotional Focus and Rating, $F(1$, $7111.1)=4.76, p=.029$. Specifically, SDO was more strongly related to how good the participants themselves felt, $b=2.55,95 \% \mathrm{Cl}[0.29,2.14]$, compared to how good participants believed the targets felt, on average, $b=1.21,95 \% \mathrm{Cl}[0.29,2.14]$ 
$(t(1201.10)=-1.97, p=.049)$. Note, however, this result was qualified by the significant five-way interaction with Target Status and Target Cooperation (see below).

Did the status and cooperation of the targets moderate participants' empathy and schadenfreude?

We predicted a significant interaction between Emotional Focus, Rating, Target Status, and Target Cooperation but it was not significant, $F(1,7111.12)=1.99, p=.158$. However, pairwise comparisons between the amount of empathy and schadenfreude felt towards the four SCM targets supported our hypotheses (Figure 1). Participants felt the most empathy towards the high-cooperation/low-status targets $(M=69.44)$, and the most schadenfreude towards the low-cooperation/high-status targets $(M=34.87)$, compared to all other targets. In fact, the amount of empathy and schadenfreude felt was significantly different for all targets, ts range between $2.65-16.79$, ps $<.041$. Again, this result was qualified by the significant five-way interaction. 


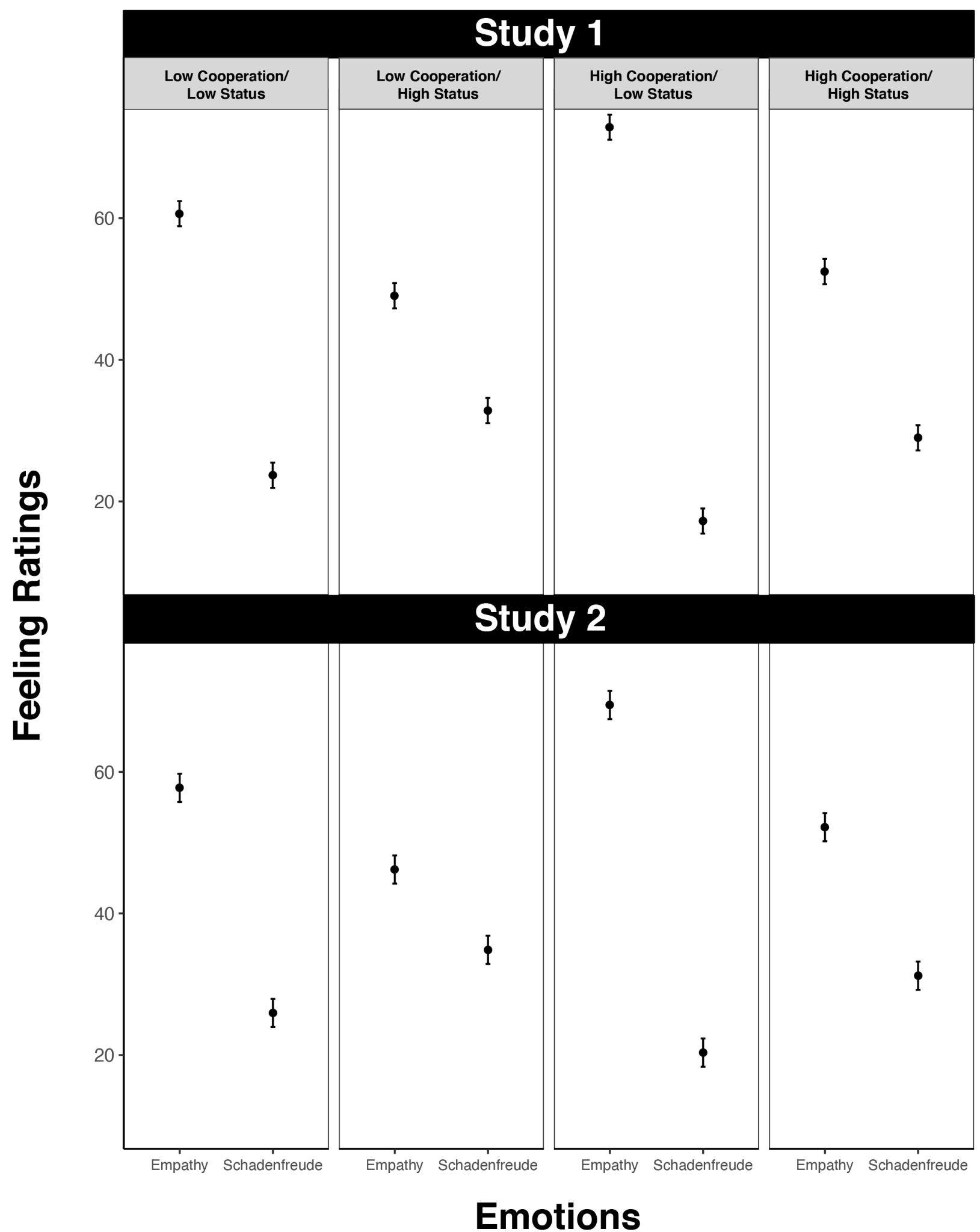

Figure 1: The amount of empathy and schadenfreude felt towards each target in Studies 


\section{Did SDO moderate how much empathy and schadenfreude participants felt towards high- and low-status targets (and could that be explained by differential attribution of emotion)?}

We expected a significant interaction between Emotional Focus, Rating, Target Status, and SDO such that in the Self-Focus condition, SDO would predict decreased empathy and increased schadenfreude for the low-status targets. The four-way interaction was not significant, $F(1,7111.1)=2.59, p=.108$, but it was qualified by a significant five-way interaction, $F(1,7111.1)=5.92, p=.015$ (Figure 2). To investigate this interaction, we compared how SDO moderated "how good" and "how bad" participants rated the target's feelings and their own feelings for each of the four SCM targets and controlled for multiple comparisons.

Starting first within the Target-Focus condition: Examining the slopes for significance, only two of the SDO slopes within the Target-Focus condition had 95\% confidence intervals that excluded zero: how good $(b=2.22,95 \% \mathrm{Cl}[0.54,3.90])$ and how bad $(b=-2.34,95 \% \mathrm{CI}[-4.02,-0.67])$ participants thought the low-cooperation/high status-targets felt. As SDO increased, participants thought the low-cooperation/highstatus targets felt less bad and more good about the negative things that happened to them. Said another way, emotion attribution did not vary as a function of SDO except for low-cooperation/high-status targets. Thus, any effects of SDO on empathy and schadenfreude beyond this could not be explained by differential attribution of emotion.

Next, we examined relationships in the Self-Focus condition, starting with the amount of schadenfreude felt by participants. The relationship between SDO and schadenfreude was not moderated by target. Specifically, SDO positively predicted 
feeling schadenfreude for all targets $\left(b_{\text {high-cooperation/low-status }}=1.97,95 \% \mathrm{CI}[0.23,3.70]\right.$;

$b_{\text {high-cooperation/high-status }}=2.36,95 \% \mathrm{CI}[0.62,4.09] ; b_{\text {low-cooperation/high-status }}=2.37$, $\left.95 \% \mathrm{Cl}[0.64,4.11] ; b_{\text {low-cooperation/low-status }}=3.51,95 \% \mathrm{CI}[1.77,5.24]\right)$. Last, we compared the amount of empathy felt by participants. The relationship between SDO and empathy did depend on target, such that higher levels of SDO were associated with less empathy only towards low-cooperation/low-status targets $(b=-3.65,95 \% \mathrm{CI}[-5.39,-1.92])$, as the 95\% confidence interval for the other targets contained zero. Furthermore, the negative relationship between SDO and empathy for low-cooperation/low-status targets was significantly more negative than the relationship between SDO and empathy for lowcooperation/high-status targets $(b=0.19,95 \% \mathrm{Cl}[-1.54,1.93]), t(7111)=-3.19, p=$ .008 , and marginally more negative than the relationship between SDO and empathy for high-cooperation/low-status targets $(b=-0.88,95 \% \mathrm{Cl}[-2.61,0.86]), t(7111)=-2.30, p=$ .097 and high-cooperation/high-status targets $(b=-0.83,95 \% \mathrm{Cl}[-2.57,0.90]), t(7111)=$ $-2.34, p=.089$. 


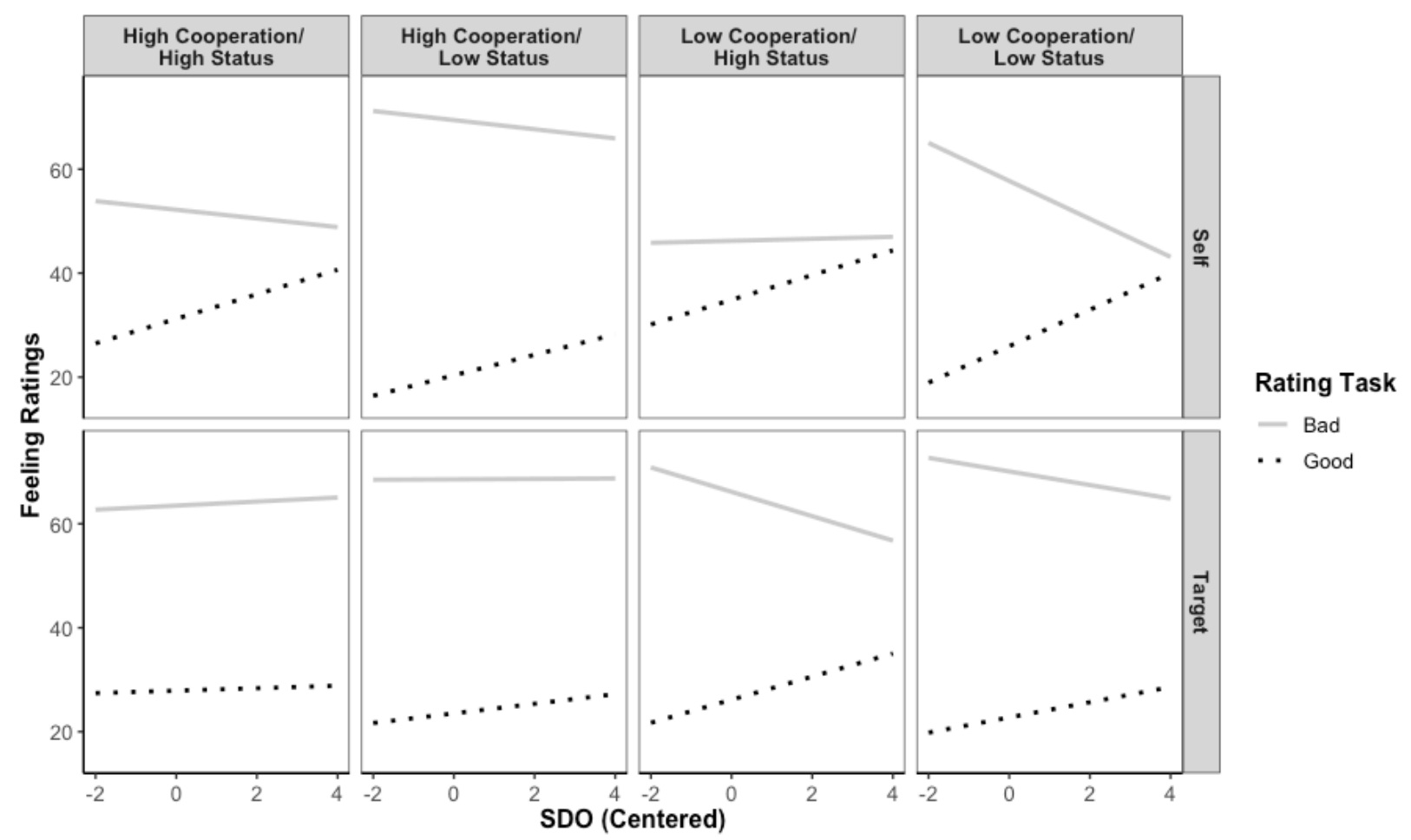

Figure 2: The effect of Rating, SDO, Target Cooperation, Target Status, and Emotional Focus on predicted Feeling Ratings in Study 1

\section{Discussion}

We tested whether the relationship between SDO and (counter-)empathy simply reflects a tendency among high-SDO individuals to ascribe fewer extreme emotions to others (which could then inform their own emotional responses). For three out of four targets (low-cooperation/low-status, high-cooperation/low status, and highcooperation/high status), SDO was not significantly related to how participants believed the target felt, suggesting that social dominants generally make the same emotional forecasts as egalitarians. However, SDO was related to how people themselves felt. Specifically, SDO was associated with increased schadenfreude towards all targets as well as reduced empathy towards low-cooperation/low-status targets (e.g., homeless people). 


\section{Study 2: The relationship between SDO and the desire to feel empathy and schadenfreude}

In Study 2, we (i) replicated self-ratings in Study 1, and (ii) tested if SDO related to the desire to feel empathy and schadenfreude. As previous work on political ideology and empathic emotions suggest (Hasson et al., 2018), individuals can desire to feel different emotions than they actually feel in the moment. There is a possibility that levels of SDO is more directly associated with the desired emotion people want to have in response to high- and low-status individuals' misfortunes in addition to the emotions the actually feel.

We had three main hypotheses. First, as in Study 1, we expected that participants reporting their actual emotions (i.e., the Actual condition) would report the greatest amount of empathy for the high-cooperation/low-status targets and the greatest amount of schadenfreude for the low-cooperation/high-status targets. Second, we expected this pattern to be moderated by SDO and target such that high SDO would be associated with feeling increased levels of schadenfreude towards all targets while predicting decreased levels of empathy specifically towards low-cooperation targets. Finally, we expected SDO to relate to a decreased desire (i.e., in the Want condition) to feel empathy towards all targets, but for the level of desire to depend on the target.

\section{Methods}

\section{Participants}

We based our sample size estimates on Study 1. Using the SIMR package, we calculated the sample size needed to achieve $80 \%$ power to detect the five-way interaction we found, (700 subjects were needed to detect an effect of $b=.51$ at $82.4 \%$ 
power) and increased it by 100 to allow for attrition. We paid a total of 844 participants from Amazon Mechanical Turk using Turk Prime as a recruitment platform, restricting our potential sample to those who were 18 years or older, residing in the United States, and had completed 500 or fewer hits. We excluded 103 participants who failed our attention check embedded within the Emotion Rating task. After exclusions we had 741 participants: 380 in the Actual condition and 361 in the Want condition, which wasn't significantly different by condition, $\chi^{2}=0.487, p=.485$. We pre-registered that we needed above 350 subjects in each condition, which we satisfied without needing to run additional subjects. 516 subjects self-identified as White and 432 as women $\left(M_{\text {age }}=\right.$ $34.81, S D=16.11)$.

\section{Procedure}

Participants who passed the initial attention check completed the same Emotion Rating task as in Study 1, but participants were either randomly assigned to indicate the emotions they actually felt (Self-Focus in Study 1; labeled 'Actual' in Study 2) or newly, the emotions they wanted to feel ('Want'). The exact prompts read: "If you saw this scenario in real life, how good/bad would it make you feel?" in the Actual condition and "If you saw this scenario in real life, how good/bad would you *want to feel?" in the Want condition. The rest of the study proceeded as in Study 1 , and $\mathrm{SDO}_{7}$ was again reliable $^{2}, \alpha=.85, M=2.63 ; S D=1.12$.

\section{Analyses}

\footnotetext{
2 We tested whether SDO varied as a function of the Emotional Judgement manipulation. It did not, $F(1$, $727)=0.2, p=.656$. However, SDO did vary as a function of counterbalancing with the stereotype content model questions. Specifically, participants had higher levels of SDO when the stereotype content model questions went first $(M=2.72)$ than when the SCM questions went second $(M=2.55), F(1,727)=$ 4.04, $p=.045, d=-0.15$.
} 
We again pre-registered our data analysis plan in which we ran a multilevel hierarchical model in $R$ where emotion ratings were predicted by the five-way interaction between Judgment (Actual, Want), Target Cooperation (Low, High), Target Status (Low, High), Rating ("how good", "how bad"), and SDO (mean-centered), including a participant random effect. All variables were within-subjects except for Judgment, which was between-subjects. The reference categories were "Actual", High Cooperation, High Status, and "How Bad".

We built the model hierarchically, moving through the main effects model to the five-way model. At each step until the five-way model, the more complicated model was a better predictor of the data. The marginal $R^{2}$ of the five-way model was .354 (the conditional $R^{2}=.406$ ). We pre-registered that we would report statistics from the fully saturated model, which is what we do below. We also conducted sensitivity analyses: With $80 \%$ power we could detect an effect size as small as $b=.55$ for the five-way interaction and $b=.54$ for the four-way interaction based on 1000 simulations.

\section{Results}

Did the status and cooperation of the targets moderate participants' empathy and counter-empathy?

The three-way interaction between Rating, Target Cooperation, and Target Status was significant, $F(1,10905.05)=27.69, p<.001$. In line with our hypotheses, participants felt the most empathy for high-cooperation/low-status targets $(M=71.46)$ and the most schadenfreude for low-cooperation/high-status targets $(M=33.68)$, ts ranged from $4.83-26.86, p s<.001$, ds ranging from $0.18-0.87$ (Figure 1 ). 


\section{Did SDO moderate how much empathy and schadenfreude participants}

\section{felt/wanted to feel in general?}

There was a significant three-way interaction between Judgement, Rating, and SDO, $F(1,10905.05)=4.17, p=.041$. SDO correlated with actual $(b=1.41$, $95 \% \mathrm{Cl}[0.48,2.35])$ and desired $(b=1.62,95 \% \mathrm{CI}[0.61,2.63])$ schadenfreude as well as with desired empathy $(b=-1.92,95 \% \mathrm{CI}[-2.93,-0.91])$, but not with actual empathy $(b=-$ $0.57,95 \% \mathrm{Cl}[-1.50,0.36])$. There was no difference in how much schadenfreude participants actually felt versus how much they desired to feel as a function of SDO, $t(1441.97)=-0.29, p=.771$, but SDO was marginally more negatively related to the desire to feel empathy compared to how much empathy was actually felt, $t(1441.97)=-$ $1.93, p=.054$. However, this effect was qualified by higher-order interactions.

Did SDO moderate how much empathy and schadenfreude participants felt versus wanted to feel towards high- and low-status targets?

Although the five-way interaction was not significant, $F(1,10905.05)=2.12, p=$ .146 , there was a significant four-way interaction between Rating, Target Cooperation, Target Status, and SDO, $F(1,1.0905 .05)=37.12, p<.001$ (Figure 3). This indicates that there was not a significant difference between how participants wanted to feel versus what they actually felt.

Examining SDO slopes within the Actual condition, we replicated Study 1. SDO negatively predicted empathy only for low-cooperation/low-status targets $(b=-4.19$, $95 \% \mathrm{Cl}[-5.34,-3.05])$, but not for low-cooperation/high-status $(b=0.73,95 \% \mathrm{Cl}[-0.41$, $1.87])$, high-cooperation/low-status $(b=-0.72,95 \% \mathrm{CI}[-1.86,0.42])$, or highcooperation/high-status $(b=-0.79,95 \% \mathrm{Cl}[-1.93,0.36])$ targets. Deviating slightly from 
Study 1 , SDO was again positively related to schadenfreude for all targets ( $b_{\text {low- }}$ cooperation/low-status $=3.51,95 \% \mathrm{CI}[2.37,4.65] ; b_{\text {high-cooperation/-low-status }}=1.55,95 \% \mathrm{CI}[0.41$, $2.69]$; $\left.b_{\text {high-cooperation/high-status }}=1.66,95 \% \mathrm{Cl}[0.52,2.80]\right)$ with the exception of the lowcooperation/high-status targets $(b=-0.66,95 \% \mathrm{CI}[-1.80,0.49])$. In sum, SDO was negatively associated with empathy and positively associated with schadenfreude for low-cooperation/low-status targets, positively associated with schadenfreude for both high-cooperation targets, and wasn't associated with either empathy or schadenfreude for low-cooperation/high-status targets.

Directly comparing slopes: the relationship between SDO and empathy for the low-cooperation/low-status targets was significantly more negative than all other targets (ts greater than $4.40, p s<.001$ ), which were not significantly different from one another (ts less than $2.00, p s>.20$ ). Unlike Study 1 , there were differences in the relationship between SDO and schadenfreude by target as well. The relationship between SDO and schadenfreude for the low-cooperation/low-status targets was significantly more positive than for high-cooperation/low-status and low-cooperation/high-status (and marginally more positive for high-cooperation/high-status targets; $t s \geq 2.43, p s \leq .071$ ), while the relationship between SDO and schadenfreude for low-cooperation/high-status targets was significantly more negative than the high-cooperation targets ( $t \leq 2.90, p s \leq$ $.020)$. 


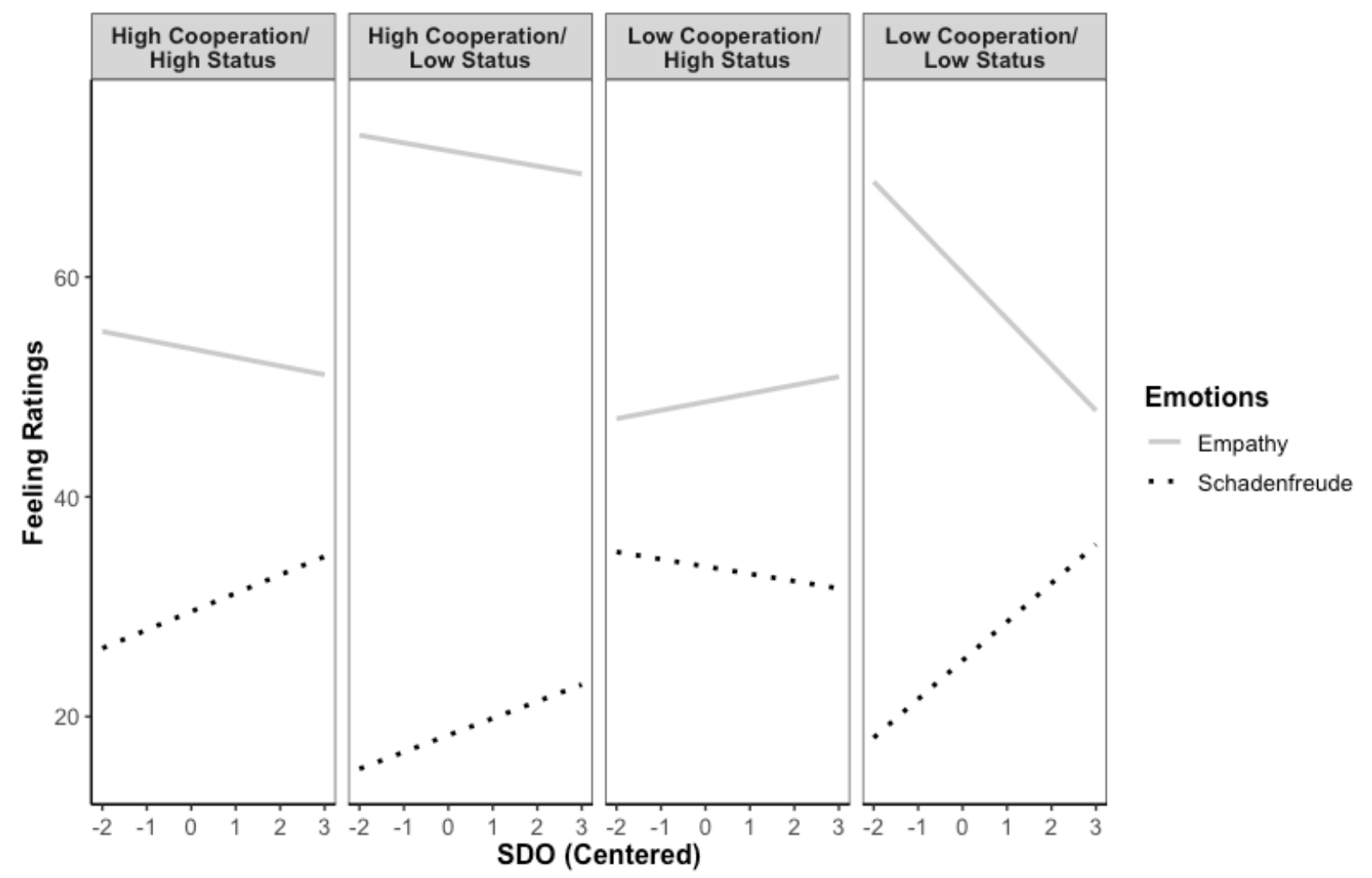

Figure 3: The effect of SDO, Target Cooperation, Target Status, and Rating (collapsing across Judgement) on predicted feeling ratings in Study 2

\section{Discussion}

Study 2 extended the findings of Study 1 by asking whether individuals higher in SDO desire to feel reduced empathy and increased counter-empathy in general but especially towards low-cooperation/low-status targets. There was not a significant fiveway interaction which suggests that there was not a significant difference between how much (counter-)empathy participants wanted to feel versus how much they actually felt as a function of SDO and target characteristics. In short, SDO related to both actual and desired levels of emotional intensity.

Study 3: The relationship between SDO and the choice to feel empathy and schadenfreude 
One obvious limitation of the previous study is that we indexed desire via selfreport and that our effect is based on a null finding. We could have not found a difference between actual emotions and desired emotions because of how we worded the questions, although the conditions were between subjects, or that participants had a hard time indicating what they would want to feel versus actually feel in a hypothetical situation. While we believe that SDO is associated with both actual and desired feelings, in Study 3 we employed a modified empathic selection task (Cameron et al., 2019) to more directly study motivation separate from actual feelings. Participants were given the ability to choose what emotion they wanted to feel (e.g., choosing between empathy and schadenfreude) on each trial, assessing whether SDO is related to situation selection, a way to regulate emotion expression (Zaki, 2014). If SDO's relationship with empathy and schadenfreude is motivated, we would expect those high in SDO to specifically avoid situations to feel empathy and opt-into situations that allow them to feel schadenfreude, especially towards low-status groups.

We limited the targets to just low-cooperation targets, because SDO most strongly moderated empathic and counter-empathic responses to those targets, and randomly assigned participants to respond to either low- or high-status targets to reduce cross-over effects across target types. We hypothesized that participants, on average, would avoid feeling empathy in general (Cameron et al., 2019), but would choose empathy for low-cooperation/low-status targets more than low-cooperation/high-status targets. However, we expected SDO to moderate both responses such that as SDO increased, participants would be less likely to choose to feel empathy (compared to 
either schadenfreude or control) in general, but especially for low-cooperation/lowstatus targets relative to low-cooperation/high-status targets.

\section{Methods}

\section{Participants}

We determined sample size using Study 4 in the work by Cameron and colleagues (2019). They had a total of 193 subjects for $80 \%$ power. Given that we reduced the number of repeated measures, included a second alternative choice to empathy (i.e., schadenfreude) and included SDO as a continuous predictor, we increased our sample size to 500 . This sample size also allowed us to have 250 subjects in each condition so that correlations could stabilize.

We recruited a total of 669 participants from Amazon Mechanical Turk using Cloud Research ${ }^{3}$ as a recruitment platform, with the same sample restrictions as Study 1. We preregistered that we would continue to recruit people until we had at least 500 useable participants. After excluding participants who failed the attention check embedded in the Empathy Selection task, we had fewer than 500 participants. Thus, we continued to recruit until the final sample was 507 (247 in the low-status condition and 260 in the high-status condition, $\left.\chi^{2}=0.333, p=.564\right)$. The sample was composed of 365 self-identified White people and 314 women $\left(M_{\text {age }}=34.7, S D=10.44\right)$.

\section{Measures}

Empathy Selection Task. This task consisted of a series of 21 trials in which participants saw a person and three decks of cards that corresponded to different set of instructions regarding how to respond to a negative event that happened to the person.

\footnotetext{
${ }^{3}$ During the production of this manuscript TurkPrime renamed itself Cloud Research.
} 
Before starting the task, we described the three decks to participants, which included an EMPATHY deck in which we asked participants to tell us "how bad you feel because of the person's misfortune", a SCHADENFREUDE deck in which we asked participants to tell us "how good you feel because of the person's misfortune" and a TARGET-FEELS deck in which we asked participants to tell us "how you think the person in the story feels about the negative event that happened to them." After we explained the task to participants, they received a series of multiple-choice comprehension checks in which they could not move forward until they chose the correct answer regarding what they were supposed to do if they chose a particular deck.

Participants then started the Empathy Selection task. On each trial participants saw a randomly assigned name and a descriptor that either made the person a lowcooperation/low-status target (i.e., "a homeless man" or a "drug addict") or a lowcooperation/high-status target (i.e., "an investment banker" or a "venture capitalist"). Participants made a choice regarding what emotion they wanted to feel (or not in the case of the TARGET-FEELS deck) and then saw a randomly assigned mildly negative story that ostensibly happened to the target. We reminded participants what they were supposed to do once they made their choice, and participants indicated how bad they felt on a 100-point slider $(0=$ not at all bad, $100=$ extremely bad $)$ if they chose the EMPATHY deck, how good they felt on a 0-100 point slider $(0=$ not at all good, $100=$ extremely good) if they chose the SCHADENFREUDE deck, and how bad the [TARGET NAME] felt on a 0-100 point slider $(0=$ not at all bad, $100=$ extremely bad $)$ if they chose the TARGET-FEELS deck. The order of the deck presentation was randomized on the trial level. Participants had 10 trials for each target description (e.g., 
10 for homeless; 10 for drug addict) and one attention check trial always administered at trial number 12 in which participants had to again move the slider all the way to the left (zero). Because participants didn't receive numeric feedback regarding the slider's position, we included participants that moved the slider to 5 or below.

Social Dominance Scale. We used the full 16-item $\mathrm{SDO}_{7}$ scale to measure social dominance and it was reliable, $\alpha=.92, M=2.51, S D=1.09$.

Task Demand. We asked participants to indicate how demanding they saw each deck on a scale from 1 (Very Demanding) - 7 (Very Easy) as a way of corroborating previous work on how cognitively demanding people see empathy. Participants saw the EMPATHY deck as the easiest $(M=4.69, S D=1.47)$, followed by the TARGET-FEELS deck, $(M=4.48, S D=1.35)$ and the SCHADENFREUDE deck, $(M=3.72, S D=1.7)$. Directly comparing the decks, participants believed the EMPATHY deck was significantly easier than both the SCHADENFREUDE, $t(505)=10.41, p<.001, d=$ 0.61 , and TARGET-FEELS decks, $t(505)=3.31, p=.001, d=0.15$. Participants also believed the TARGET-FEELS deck was significantly easier than the SCHADENFREUDE deck, $t(505)=-8.72, p<.001, d=-0.50$.

\section{Procedures}

After participants passed the screener described in Study 1, they completed the Empathy Selection task. We randomly assigned participants to read about lowcooperation/low-status targets or low-cooperation/high-status targets, making Target a between-subjects factor unlike Studies 1 and 2. After participants completed the Empathy Selection task, they filled out the $\mathrm{SDO}_{7}$ scale, indicated how mentally demanding the different kinds of trials were, and filled out brief demographics. 


\section{Analyses}

We pre-registered that we would analyze the choice data ${ }^{4}$ using a multilevel logistic regression model in $R$ where choice was predicted by the three-way interaction between Target (Low-Cooperation/Low-Status, Low-Cooperation/High-Status), Deck Options (EMPATHY, SCHADENFREUDE,TARGET-FEELS), and SDO (meancentered). However, our data actually required a multinomial multilevel regression rather than a multilevel logistic regression as there were three possible options for participants to choose on each trial. We helmert-coded Deck Options such that the Empathy choice was compared to Schadenfreude and Target-Feels combined, then the choice to pick Schadenfreude was compared to Target-Feels. We effects-coded Target such that low-cooperation/low-status was the reference category and included a participant random intercept. Finally, we conducted Wald tests to assess the overall impact of any effects that contained Deck Options as a variable of interest.

We calculated that with $80 \%$ power we could detect an effect size as small as $b=$ .08 for the three-way interaction comparing Schadenfreude to Target-Feels and $b=.045$ for the three-way interaction comparing Empathy to Schadenfreude and Target-Feels combined.

\section{Results}

\section{Did participants, on average, avoid feeling empathy?}

No. There was a main effect of Deck Options, $X^{2}(2, N=507)=388.51, p<.001$ which was significant for both of our contrasts. Specifically, the comparison between

\footnotetext{
${ }^{4}$ We did not analyze participants emotional responses on the 100-point sliders because of the nature of the task; we did not have random assignment regarding whether participants reported their levels of empathy or schadenfreude and thus focused on their deck choices.
} 
choosing the EMPATHY deck or anything else was significant, $b=0.21, p<.001$, as well as the comparison between choosing the SCHADENFREUDE deck compared to the TARGET-FEELS deck, $b=-0.25, p<.001$. The odds of choosing the EMPATHY deck were 1.232 times greater $(95 \% \mathrm{CI}[1.203,1.262])$ than the odds of choosing anything else. The odds of choosing the SCHADENFREUDE deck were 0.78 (95\% $\mathrm{CI}[0.747,0.815])$ as large as the odds of choosing the TARGET-FEELS deck. Thus, we did not find evidence in support of the hypothesis that individuals preferred to avoid feeling empathy. Instead, participants chose to feel empathy most often. On average, participants chose the EMPATHY deck on $39.45 \%$ of trials, SCHADENFREUDE on $26.73 \%$ of trials, and TARGET-FEELS on $33.83 \%$ of trials.

\section{Did participants, on average, choose to feel empathy more often for low- cooperation/low-status versus low-cooperation/high-status targets?}

There was a significant interaction between Target and Deck Option, $\chi^{2}(2, N=$ $507)=86.07, p<.001$. This overall interaction effect was also significant for each of our contrasts. The comparison between choosing the EMPATHY deck over anything else between low-cooperation/low-status and low-cooperation/high-status targets was significant, $b=-0.139, p<.001, \mathrm{OR}=0.87(95 \% \mathrm{Cl}[0.841,0.9])$ as well as the comparison between choosing the SCHADENFREUDE deck over the TARGET-FEELS deck for low-cooperation/low-status and low-cooperation/high-status targets, $b=0.148$, $p<.001, O R=1.16(95 \% \mathrm{Cl}[1.092,1.232])$. The odds of choosing the EMPATHY deck over everything else decreased by 0.87 moving from the low-cooperation/low-status target to the low-cooperation/low-status target. The odds of choosing the SCHADENFREUDE deck over the TARGET-FEELS deck increased by 1.16 moving 
from the low-cooperation/low-status target to the low-cooperation/high-status target.

Thus, our hypothesis was supported; participants chose to feel more empathy, and less schadenfreude, towards low-cooperation/low-status targets compared to lowcooperation/high-status targets.

Did SDO moderate participants' choice to feel empathy and schadenfreude in general?

There was a significant interaction between SDO and Deck Option, $\chi^{2}(2, N=$ $507)=34.27, p<.001$. However, this overall effect held for only one contrast, which was the comparison between choosing the EMPATHY deck compared to everything else, $b=-0.07, p<.001, \mathrm{OR}=0.94(95 \% \mathrm{Cl}[0.92,0.96])$. For every unit increase in SDO, the odds of choosing the EMPATHY deck over everything else decreased by

0.94. There was not a significant difference in the probability of choosing the SCHADENFREUDE deck over the TARGET-FEELS deck as a function of SDO, $b=$ $0.02, p=.287, \mathrm{OR}=1.02(95 \% \mathrm{Cl}[0.98,1.06])$. Thus, our hypothesis regarding SDO's modulation of empathic responding was partially supported. As SDO increased, participants were less likely to choose to feel empathy compared to the alternative options but not more likely to choose to feel schadenfreude compared to the control condition.

Did SDO moderate how much empathy and schadenfreude participants chose to feel towards low-cooperation/low-status and low-cooperation/high-status targets?

There was a marginal significant three-way interaction between Target, Deck Option, and SDO, $\chi^{2}(2, N=507)=4.79, p=.091$ (Figure 4). Looking at the specific 
contrasts, the first contrast was significant while the second was not. Choosing the EMPATHY deck compared to everything else as a function of SDO differed by Target, $b$ $=0.034, p=.029, \mathrm{OR}=1.035(95 \% \mathrm{Cl}[1.004,1.067])$ but there was not a significant difference between Targets in terms of choosing the SCHADENFREUDE deck compared to the TARGET-FEELS deck, $b=-0.011, p=.702$, OR $=0.989(95 \%$ $\mathrm{CI}[0.936,1.046])$. Comparing our helmert-coded contrasts, we find that the relationship between SDO and the likelihood of choosing the EMAPTHY deck versus anything else was more strongly negative for low-cooperation/low-status targets $(\beta$ : Empathy $=-0.12$; Schadenfreude $=0.10 ;$ Target-Feels $=0.05)$ than for low-cooperation/high-status targets $(\beta$ : Empathy $=-0.06$; Schadenfreude $=0.04 ;$ Target-Feels $=0.02)$, Diff of Diff $=$ $0.21, z=2.18, p=.029$. However, there was no difference in the choice between SCHADENFREUDE compared to the TARGET-FEELS deck as a function of SDO and Target. 


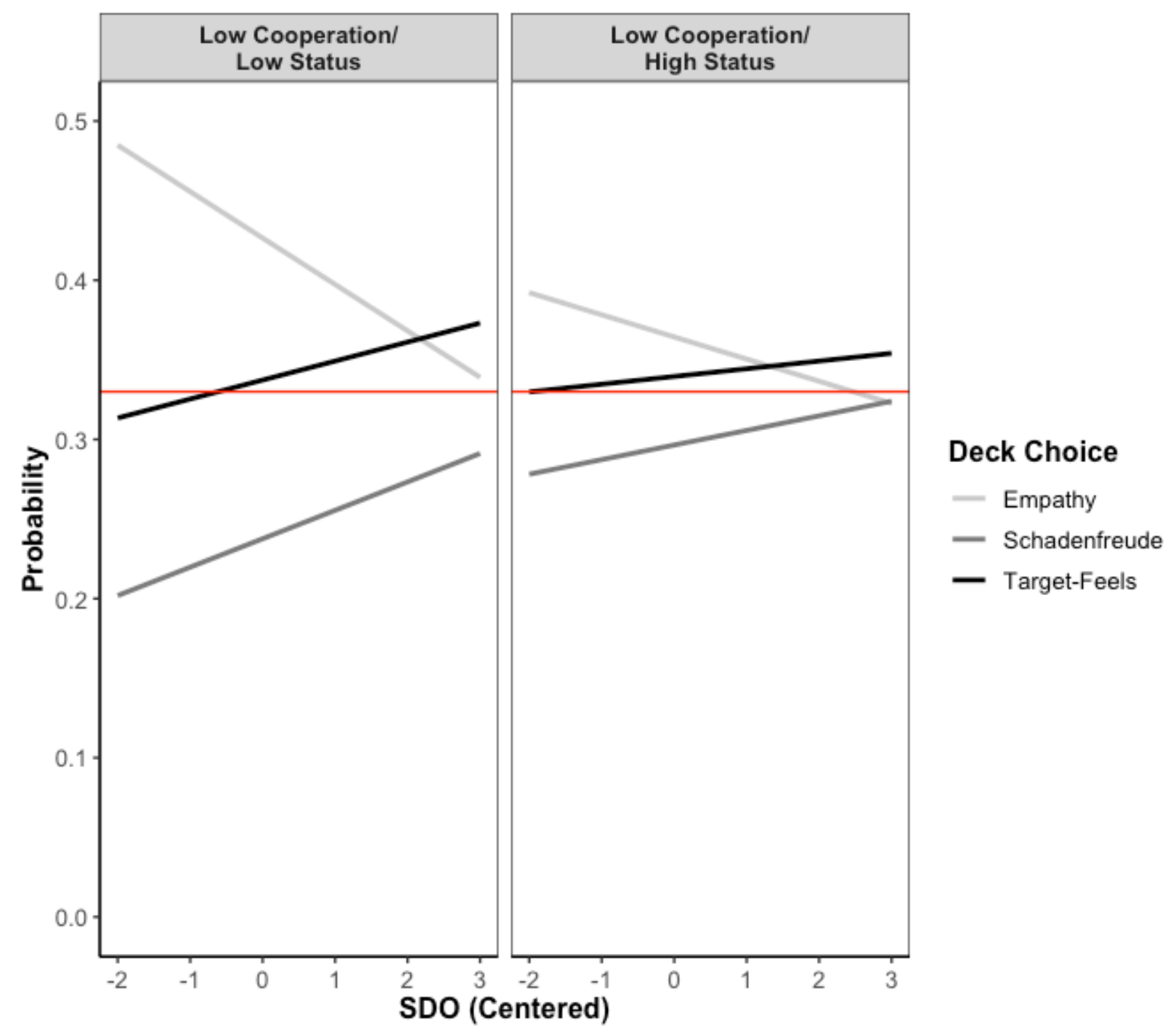

Figure 4: The effect of SDO, Target, and Deck Options on the probability of deck choices. The red line indicates chance responding at .33

\section{Discussion}

Study 3 assessed the relationship between SDO and the choice to feel less empathy and more schadenfreude. In contrast to Cameron et al. (2019), participants in our study found the Empathy task the easiest to do and correspondingly chose the Empathy deck most frequently. However, even in a situation in which empathy was the preferred emotion of participants on average, we still found that SDO was negatively 
related to participants' likelihood of choosing to feel empathy in general, and that this was more the case for low-cooperation/low-status versus low-cooperation/high-status targets. We did not find support for our hypotheses regarding schadenfreude. However, SDO was significantly positively related to the likelihood of choosing to feel schadenfreude for the low-cooperation/low-status targets, $b=0.10,95 \% \mathrm{Cl}[0.04,0.16]$ (but not the low-cooperation/high-status targets, $b=0.04,95 \% \mathrm{Cl}[-0.01,0.10]$ ). Furthermore, the schadenfreude deck was seen as the most demanding, yet those with higher levels of SDO chose that deck more often than those with lower levels of SDO. Indeed, SDO was minimally correlated with deck demand, with the highest correlation being with the Empathy deck demand, $r=-0.10, p=.0211$.

\section{General Discussion}

In this paper we tested whether (counter-)empathic emotions elicited in intergroup contexts not only relate to levels of SDO but are motivated by them. We found that while SDO didn't systematically associate with attribution of emotion to others, SDO did relate to feeling reduced empathy and increased schadenfreude, especially towards those who are disadvantaged in society. Moreover, SDO simultaneously related to individuals' desires and choices to avoid empathy for disadvantaged targets, and less so for schadenfreude.

The research presented here comports with other work examining the influence of SDO on empathy. For example, previous work on SDO finds a positive relationship with feeling empathy for advantaged targets, albeit inconsistently, and a negative relationship with feeling empathy for disadvantaged targets (Lucas \& Kteily, 2018). We did not replicate their results by which SDO more strongly related to feeling empathy 
(positively or negatively) for low-cooperation/high-status targets - the targets that most closely resemble Lucas \& Kteily's operationalization of advantaged targets. However, in line with their findings, SDO was strongly related to feeling reduced empathy for lowcooperation/low-status targets, who are more disadvantaged in society than lowcooperation/high-status targets.

Our findings highlight many avenues for future research. We discuss two here. The first is the role of cooperation, or warmth perceptions in social dominants' affective and cognitive reactions to social groups. In this work we found that for those groups high in cooperation, SDO's relationship with empathy and schadenfreude was attenuated, suggesting that targets seen with high cooperation are not seen as either a threat or a boon to hierarchical processes. Instead, the strongest relationships between SDO and empathic and counter-empathic responding were reserved for those low in cooperation, and in particular, those also low in status. While we didn't hypothesize about the impact of cooperation on these processes, these findings are in line with theorizing around SDO more broadly. As SDO reflects a competitive view of the world, it is consistent that those who are seen as high in warmth, or cooperation, are immune from processes that usually track with SDO. Future research should more directly investigate how cooperation perceptions align with social dominants' feelings of empathy and schadenfreude.

Second, it is unclear whether SDO modulates empathic responding at all stages of the motivational pathway. We've demonstrated that SDO is related to the choice to approach or avoid situations in which the emotion that is likely to be elicited is known. However, there are other ways to regulate emotions, such as attention modulation (i.e., 
the choice to attend to various elements of the situation that might evoke empathy, or not) and appraisal (i.e., changing the appraisal of the intensity of the target's emotion or the meaning of the target's emotions; Zaki, 2014). Research on SDO and attention to inequality (Kteily et al., 2017) suggests that attention modulation is a strategy individuals higher in SDO also potentially use to regulate their empathic responding. The fact that SDO related to beliefs that low-cooperation/high-status targets felt less bad and more good about negative events that occur to them in Study 1 suggests that SDO could be related to appraisals of the meaning of others' experiences. For example, social dominants could believe to a greater degree that negative events happening to people are less meaningful and thus do not deserve as intense of an empathic response. Social dominants could also believe that negative events are more meaningful and yet feel reduced empathy and increased counter-empathy intentionally in response. More research is needed to elucidate situations in which SDO would be associated with decreased or increased event appraisals.

There are some limitations to our findings, including the fact that we did not manipulate levels of SDO and showed a corresponding shift in desire or choice to feel empathy and schadenfreude. While manipulating SDO is difficult, future work should continue to build the causal argument for SDO and emotional reactions. Additionally, this research purposefully measured affective reactions in social dominants towards third-party targets. There is an outstanding question whether SDO motivates empathic and counter-empathic responses within intergroup dynamics specifically. We believe this is the case, but it is an empirical question. Finally, we also didn't fully support hypotheses around SDO and schadenfreude. While this could be due to SDO not being 
as related to the motivations to feel schadenfreude, we believe it's more likely that schadenfreude is simply harder to capture than empathy. Future studies should use subtler measures of schadenfreude, such as physiological responses (Cikara \& Fiske, 2012).

\section{Conclusion}

The current research supports the assertion that SDO can lead individuals to engage in motivated emotional responses. This work expands our understanding of the motivational role of ideologies in emotional reactions in intergroup contexts. 


\section{References}

Bartoń, K. (2018). MuMIn: Multi-model inference. https://cran.rproject.org/web/packages/MuMIn/MuMIn.pdf

Cameron, C. D., Hutcherson, C. A., Ferguson, A. M., Scheffer, J. A., Hadjiandreou, E., \& Inzlicht, M. (2019). Empathy is hard work: People choose to avoid empathy because of its cognitive costs. Journal of Experimental Psychology: General, 148(6), 962-976. https://doi.org/10.1037/xge0000595

Cikara, M., Bruneau, E. G., \& Saxe, R. R. (2011). Us and them: Intergroup failures of empathy. Current Directions in Psychological Science, 20(3), 149-153. https://doi.org/10.1177/0963721411408713

Cikara, M., Bruneau, E., Van Bavel, J. J., \& Saxe, R. (2014). Their pain gives us pleasure: How intergroup dynamics shape empathic failures and counter-empathic responses. Journal of Experimental Social Psychology, 55, 110-125. https://doi.org/10.1016/j.jesp.2014.06.007

Cikara, M., \& Fiske, S. T. (2011). Bounded Empathy: Neural Responses to Outgroup Targets' (Mis)fortunes. Journal of Cognitive Neuroscience, 23(12), 3791-3803. https://doi.org/10.1162/jocn_a_00069

Cikara, M., \& Fiske, S. T. (2012). Stereotypes and schadenfreude: Affective and physiological markers of pleasure at outgroup misfortunes. Social Psychological and Personality Science, 3(1), 63-71. https://doi.org/10.1177/1948550611409245

Cikara, M., \& Fiske, S. T. (2013). Their pain, our pleasure: stereotype content and schadenfreude. Annals of the New York Academy of Sciences, 1299(1), 52-59. https://doi.org/10.1111/nyas.12179 
Cottrell, C. A., \& Neuberg, S. L. (2005). Different Emotional Reactions to Different Groups: A Sociofunctional Threat-Based Approach to "Prejudice". Journal of Personality and Social Psychology, 88(5), 770-789. https://doi.org/10.1037/00223514.88.5.770

Craig, M. A., \& Richeson, J. A. (2014). Not in my backyard! authoritarianism, social dominance orientation, and support for strict immigration policies at home and abroad. Political Psychology, 35(3), 417-429. https://doi.org/10.1111/pops.12078

Cuddy, A. J. C., Fiske, S. T., \& Glick, P. (2008). Warmth and competence as universal dimensions of social perception: The stereotype content model and the BIAS map. In Advances in Experimental Social Psychology (Vol. 40, pp. 61-149). https://doi.org/10.1016/S0065-2601(07)00002-0

Dovidio, J. F., Johnson, J. D., Gaertner, S. L., Pearson, A. R., Saguy, T., \& AshburnNardo, L. (2010). Empathy and intergroup relations. In M. Mikulincer \& P. R. Shaver (Eds.), Prosocial motives, emotions, and behavior: The better angels of our nature. (pp. 393-408). American Psychological Association. https://doi.org/10.1037/12061-020

Federico, C. M., \& Sidanius, J. (2002). Racism, ideology, and affirmative action revisited: The antecedents and consequences of "principled objections" to affirmative action. Journal of Personality and Social Psychology, 82(4), 488-501. https://doi.org/10.1037//0022-3514.82.4.488

Fiske, S. T., Cuddy, A. J. C., Glick, P., \& Xu, J. (2002). A model of (often mixed) stereotype content: Competence and warmth respectively follow from perceived status and competition. Journal of Personality and Social Psychology, 82(6), 878- 
902. https://doi.org/10.1037//0022-3514.82.6.878

Fraser, G., Osborne, D., \& Sibley, C. G. (2015). "We want you in the Workplace, but only in a Skirt!" Social Dominance Orientation, Gender-Based Affirmative Action and the Moderating Role of Benevolent Sexism. Sex Roles, 73(5-6), 231-244. https://doi.org/10.1007/s11199-015-0515-8

Goudarzi, S., Pliskin, R., Jost, J. T., \& Knowles, E. (2019). Economic System Justification Predicts Muted Emotional Responses to Inequality. In Nature Communications. https://doi.org/https://osf.io/up8ay

Gutiérrez, A. S., \& Unzueta, M. M. (2013). Are admissions decisions based on family ties fairer than those that consider race? Social dominance orientation and attitudes toward legacy vs. affirmative action policies. Journal of Experimental Social Psychology, 49(3), 554-558. https://doi.org/10.1016/j.jesp.2012.10.011

Haley, H., \& Sidanius, J. (2006). The positive and negative framing of affirmative action: A group dominance perspective. Personality and Social Psychology Bulletin, 32(5), 656-668. https://doi.org/10.1177/0146167205283442

Hasson, Y., Tamir, M., Brahms, K. S., Cohrs, J. C., \& Halperin, E. (2018). Are liberals and conservatives equally motivated to feel empathy toward others? Personality and Social Psychology Bulletin, 44(10), 1449-1459. https://doi.org/10.1177/0146167218769867

Hiel, A. Van, \& Mervielde, I. (2005). Authoritarianism and social dominance orientation: Relationships with various forms of racism. Journal of Applied Social Psychology, 35(11), 2323-2344. https://doi.org/10.1111/j.1559-1816.2005.tb02105.x Ho, A. K., Sidanius, J., Kteily, N., Sheehy-Skeffington, J., Pratto, F., Henkel, K. E., 
Foels, R., \& Stewart, A. L. (2015). The nature of social dominance orientation: Theorizing and measuring preferences for intergroup inequality using the new $\mathrm{SDO}_{7}$ scale. Journal of Personality and Social Psychology, 109(6), 1003-1028. https://doi.org/10.1037/pspi0000033

Ho, G. C., \& Unzueta, M. M. (2015). Antiegalitarians for affirmative action? When social dominance orientation is positively related to support for egalitarian social policies. Journal of Applied Social Psychology, 45(8), 451-460. https://doi.org/10.1111/jasp.12311

Hudson, S. T. J., Cikara, M., \& Sidanius, J. (2019). Preference for hierarchy is associated with reduced empathy and increased counter-empathy towards others, especially out-group targets. Journal of Experimental Social Psychology, 85(May), 103871. https://doi.org/10.1016/j.jesp.2019.103871

Jost, J. T., Banaji, M. R., \& Nosek, B. A. (2004). A decade of system justification theory: Accumulated evidence of conscious and unconscious bolstering of the status quo. Political Psychology, 25(6), 881-919. http://onlinelibrary.wiley.com/doi/10.1111/j.1467-9221.2004.00402.x/abstract Jost, J. T., Glaser, J., Kruglanski, A. W., \& Sulloway, F. J. (2003). Political Conservatism as Motivated Social Cognition. Psychological Bulletin, 129(3), 339-375. https://doi.org/10.1037/0033-2909.129.3.339

Kteily, N. S., Sheehy-Skeffington, J., \& Ho, A. K. (2017). Hierarchy in the eye of the beholder: (Anti-)egalitarianism shapes perceived levels of social inequality. Journal of Personality and Social Psychology, 112(1), 136-159.

https://doi.org/10.1037/pspp0000097 
Lange, J., \& Boecker, L. (2019). Schadenfreude as Social-Functional Dominance Regulator. Emotion, 19(3), 489-502. https://doi.org/10.1037/emo0000454

Lucas, B. J., \& Kteily, N. (2016). High SDO Individuals Display Moral Concern for Harm to High Status (but not Low Status) Targets. Academy of Management Proceedings, 2016(1), 12048. https://doi.org/10.5465/ambpp.2016.80

Lucas, B. J., \& Kteily, N. S. (2018). (Anti-)egalitarianism differentially predicts empathy for members of advantaged versus disadvantaged groups. Journal of Personality and Social Psychology, 114(5), 665-692. https://doi.org/10.1037/pspa0000112

Mackie, D. M., Devos, T., \& Smith, E. R. (2000). Intergroup emotions: Explaining offensive action tendencies in an intergroup context. Journal of Personality and Social Psychology, 79(4), 602-616. https://doi.org/10.1037/0022-3514.79.4.602

Nicol, A. A. M., \& Rounding, K. (2013). Alienation and empathy as mediators of the relation between social dominance orientation, right-wing authoritarianism and expressions of racism and sexism. Personality and Individual Differences, 55(3), 294-299. https://doi.org/10.1016/j.paid.2013.03.009

Porat, R., Halperin, E., \& Tamir, M. (2016). What we want is what we get: Group-based emotional preferences and conflict resolution. Journal of Personality and Social Psychology, 110(2), 167-190. https://doi.org/10.1037/pspa0000043

Pratto, F., \& Lemieux, A. F. (2001). The Psychological Ambiguity of Immigration and Its Implications for Promoting Immigration Policy. Journal of Social Issues, 57(3), 413430. https://doi.org/10.1111/0022-4537.00221

Richard, F. D., Bond, C. F., \& Stokes-Zoota, J. J. (2003). One Hundred Years of Social Psychology Quantitatively Described. Review of General Psychology, 7(4), 331- 
363. https://doi.org/10.1037/1089-2680.7.4.331

Schönbrodt, F. D., \& Perugini, M. (2013). At what sample size do correlations stabilize? Journal of Research in Personality. https://doi.org/10.1016/j.jrp.2013.05.009

Sherman, G. D., Lerner, J. S., Renshon, J., Ma-Kellams, C., \& Joel, S. (2015).

Perceiving Others' Feelings: The Importance of Personality and Social Structure. Social Psychological and Personality Science, 6(5), 559-569. https://doi.org/10.1177/1948550614567358

Sibley, C. G., Wilson, M. S., \& Duckitt, J. (2007). Antecedents of men's hostile and benevolent sexism: the dual roles of social dominance orientation and right-wing authoritarianism. Personality and Social Psychology Bulletin, 33(2), 160-172. https://doi.org/10.1177/0146167206294745

Sidanius, J., Kteily, N., Sheehy-Skeffington, J., Ho, A. K., Sibley, C., \& Duriez, B. (2013). You're inferior and not worth our concern: The interface between empathy and social dominance orientation. Journal of Personality, 81(3), 313-323. https://doi.org/10.1111/jopy.12008

Smith, R. H., \& van Dijk, W. W. (2018). Schadenfreude and Gluckschmerz. Emotion Review, 10(4), 293-304. https://doi.org/10.1177/1754073918765657

Thomsen, L., Green, E. G. T., \& Sidanius, J. (2008). We will hunt them down: How social dominance orientation and right-wing authoritarianism fuel ethnic persecution of immigrants in fundamentally different ways. Journal of Experimental Social Psychology, 44(6), 1455-1464. https://doi.org/10.1016/j.jesp.2008.06.011

Zaki, J. (2014). Empathy: A motivated account. Psychological Bulletin, 140(6), 16081647. https://doi.org/10.1037/a0037679 
